\title{
Revising Qualitative Constraint Networks: Definition and Implementation
}

\author{
Julien Hué and Matthias Westphal \\ Institut für Informatik \\ Albert-Ludwigs-Universität Freiburg \\ Georges-Köhler-Allee 52 \\ 79110 Freiburg, Germany \\ \{hue,westpham\}@informatik.uni-freiburg.de
}

\begin{abstract}
Qualitative Spatial and Temporal Reasoning is a central topic in Artificial Intelligence. In particular, it is aimed at application scenarios dealing with uncertain information and thus needs to be able to handle dynamic beliefs. This makes merging and revision of qualitative information important topics. While merging has been studied extensively, revision which describes what is happening when one learns new information about a static world has been overlooked. In this paper, we propose to fill the gap by providing two revision operations for qualitative calculi. In order to implement these operations, we give algorithms for revision and analyze the computational complexity of these problems. Finally, we present an implementation of these algorithms based on a qualitative constraint solver and provide an experimental evaluation.
\end{abstract}

\section{INTRODUCTION}

Qualitative Spatial and Temporal Reasoning (QSTR) is a research field which studies relation languages for representing information about infinitely-valued domains. For example, temporal relations between events (defined on the domain $\mathbb{Q}$ ) can be described by qualitative terms like $a$ happened before $b$ or $a$ takes place during $b$. Since spatial and temporal aspects are usually concerned with infinite, continuous domains, many computational tasks are undecidable in the general case. Thus, focusing on the qualitative relations between entities is a convenient method for crafting a calculus that can actually be used for reasoning. Qualitative information is then represented as constraint networks defined over such qualitative terms, socalled qualitative constraint networks (QCNs).

As qualitative information deals with vague and uncertain information, representing Qualitative Spatial and Temporal beliefs is an important topic for several fields in or related to Artificial Intelligence, e.g., Geographic Information Systems or robot navigation. So far, mainly the central task of deciding satisfiability of QCNs has been extensively studied. Nevertheless, many practical applications of such qualitative languages require the ability to deal with changing beliefs, i.e., if one only knows uncertain or incomplete information about the world then what happens when a more reliable piece of information about the world arises or when the world changes? The first problem is known as belief revision and the latter problem is known as belief update. For this paper, we will only concentrate on the revision problem (differences with possible treatments of update operations will be discussed in the conclusion). A good revision operation must solve conflicts between the new and the old information while saving as much as possible from the old information and respecting the new one. Such issues arise in, e.g., the context of robots learning a map of their surroundings [Wal10]. Several operators have been studied for merging QCNs (see [WD10], [CKMS09a], [CKMS10], [CKMS09b]). These merging operators are based on the distance between solutions of each source (called semantic operators) or based on the number of violated constraints (called syntactic operators). These distances in turn can either be based on a drastic distance or a more finegrained distance based on proximity of the different relations [CKS08]. To the authors knowledge, with the exception of a bachelor's thesis [Dos11] on classical revision operations (partial meet and cut revisions) for QCNs, no operations for revision of QCNs have been proposed. Moreover, in the context of QCNs, to the authors knowledge no such revision or merging operation has been implemented despite some proposals such as [WD10].

In this paper, we propose revision operations for QCNs based on the merging operations previously defined in the literature. We show that these operations behave well with respect to the AGM postulates which state how a correct revision operator must behave [AGM85]. We then provide an algorithm for revision operations of QCNs and analyze its computational complexity. We finally exhibit an implementation of these revision operations based on qualitative constraint reasoning techniques and provide an experimental study. We based this implementation on the qualitative constraint solver GQR [WWG09] since it is considered as the state-of-the-art constraint solver for QCNs and compares favorably with other possibilities such as SAT encodings [WW09].

The structure of this paper is as follows. In Section II a reminder about QCNs and revision is given. Then, a formal definition of our revision operations is provided in Section III where their formal properties are also studied. This is followed by a description of our implementation in Section IV as well as a theoretical study of its complexity. In Section $\mathrm{V}$ we then show first experimental results of our implementation. We conclude the paper in Section VI with a summary and outlook on future work. 


\section{BACKGROUND AND NOTATION}

In this section we introduce and define Qualitative Constraint Networks, some operations for merging such QCNs and the set of AGM postulates for general revision operations.

\section{A. Qualitative Spatial and Temporal Reasoning}

The work is based on the formalisms used for qualitative spatial and temporal reasoning. We define these qualitative calculi along with the representation of qualitative information in constraint networks. These standard definitions can be found in, e.g., [Ren02], [CKMS09a].

Definition 1 (Partition Scheme). Let $\mathcal{D}$ be a non-empty domain. A partition scheme is a partition of $\mathcal{D}^{2}$ into a finite set $B=\left\{b_{1}, \ldots, b_{n}\right\}$ of non-empty binary relations on $\mathcal{D}$ such that it contains the identity relation on $\mathcal{D}^{2}$, and is closed under the converse operation $\left(\forall b \in B \exists b^{\prime} \in B: b=b^{\prime-1}\right)$. The elements of $B$ are called base relations.

Base relations from a partition schemes provide an exhaustive set of qualitative terms for describing relations between entities on $\mathcal{D}$. In order to deal with uncertain and vague information, we consider unions of base relations as well. This gives us the set $2^{B}$ as the full set of qualitative terms we can use to describe relations on $\mathcal{D}$. In particular, it contains the empty set (an unsatisfiable relation) and the universal relation (which is always satisfied). We represent qualitative information in constraint networks.

Definition 2 (Qualitative Constraint Network). A Qualitative Constraint Network $(Q C N) N$ over $2^{B}$ is a pair $(V, C)$ where:

- $V$ is a finite set of variables $\left\{v_{1}, \ldots, v_{n}\right\}$ such that every variable $v_{i} \in V$ represents an entity of the domain $D$;

- $C$ is a finite set of constraints which are triplets $\left(v_{1}, R, v_{2}\right)$ where $v_{1}, v_{2} \in V$ and $R \in 2^{B}$. If $\left(v_{1}, R, v_{2}\right) \in C$ then the relation $R$ must hold between $v_{1}$ and $v_{2}$. Further, for every pair $v_{1}, v_{2} \in V$ there is exactly one such triplet.

From now on, we will denote $\left(v_{i}, R, v_{j}\right)$ by $R_{i j}$. Finally, to allow for reasoning on constraint networks we consider inference rules defined on constraints $R_{i j}$ in the network. Firstly, we have converse rules:

$$
R_{i j} \rightarrow R_{j i}^{-1}
$$

for every $R \in 2^{B}$ and pair of variables. Secondly, we have rules on every triple of relations $R, S, T \in 2^{B}$ and variables $\left(v_{i}, v_{j}, v_{k}\right)$ :

$R_{i j} \wedge S_{j k} \rightarrow T_{i k} \in\left\{b \in B \mid \exists v_{i}, v_{j}, v_{k} \in \mathcal{D}: b_{i k} \wedge R_{i j} \wedge S_{j k}\right\}$

Rule (2) expresses the set of valid descriptions involving three variables. It ensures the possible relations to be locally consistent wrt. real world instantiations.

Note that there is only a finite number of these inference rules as they are defined on $2^{B}$ and not directly $\mathcal{D}$.

Definition 3 (Qualitative Calculus). The symbols of a fixed partition scheme $B$ together with the corresponding set of inference rules (1) and (2) define an entirely symbolic formalism, a so-called qualitative calculus.
Region Connection Calculi as an Example: An important family of qualitative calculi are the Region Connection Calculi (RCC), which comprises different formalisms for reasoning about topological relations between spatially extended objects (called regions) [RCC92], [RC89], [CH01]. The theory of Region Connection Calculi is built on a binary connectedness predicate and can be cast as a first-order theory. Different fragments can be obtained depending on further distinctions being made (e.g., distinctions between interior, bounding points, or the convex hull of regions). Regions are typically interpreted as regular open (or regular closed) sets of a topological space, and we do so here as well, when we illustrate regions by simple regions in $\mathbb{R}^{2}$.

The simplest of the RCC is RCC5 [Ben94] which deals with relations that can be defined in terms of parthood of simple regions or closed disks. The language of RCC5 contains five binary relations $B=\{D R, E Q, P O, P P, P C\}$ with the following interpretation: $D R_{a b}$ means that $a$ and $b$ are completely disconnected; $E Q_{a b}$ means that $a$ and $b$ are identical; $P O_{a b}$ means that $a$ and $b$ partially overlap; $P P_{a b}$ means that $a$ is a proper part of $b ; P C_{a b}$ means that $b$ is a proper part of $a$.

When considering the border of these regions, $D R$ can be divided into $D C$ (DisConnected) and $E C$ (Externally Connected), $P P$ can be divided into $T P P$ (Tangential Proper Part) and NTPP (Non-Tangential Proper Part) and $P C$ into $T P P I$ and NTPPI. These relations are illustrated in Fig. 1.

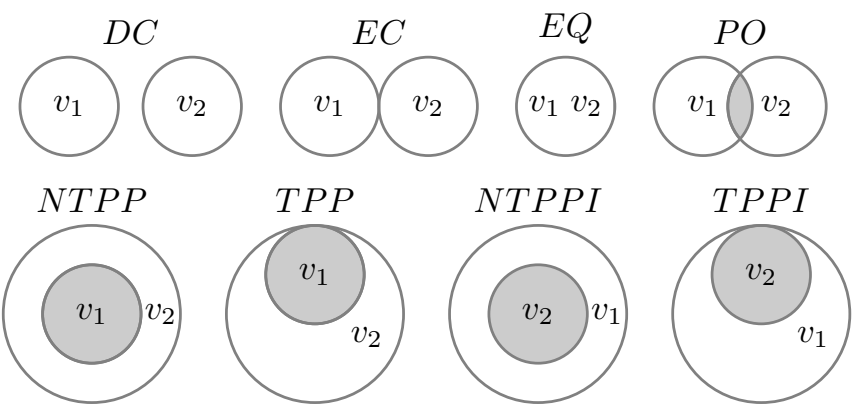

Fig. 1. Relations defined in the RCC8 calculus

Further, we recall the definition of (conceptual) neighborhood graphs. Neighborhood graphs are undirected graph aiming at representing conceptual proximity between base relations. This conceptual proximity is usually based on the number of topological transformation needed to go from one state to one another considering only two entities.

Definition 4 (Neighborhood Graph [EAT92]). A neighborhood graph of a qualitative calculus with base relations $B$ is an undirected graph with vertices as elements of $B$ and an edge between $b$ and $b^{\prime}$ iff $b$ and $b^{\prime}$ are considered to be conceptually close.

As an example, Fig. 2 depicts a possible neighborhood graph for RCC8.

Finally, with these concepts we can define the notion of scenarios and consistency of QCNs.

Definition 5 (Scenario). A scenario $s$ is a $Q C N$ in which every constraint is defined by a base relation and we cannot derive 


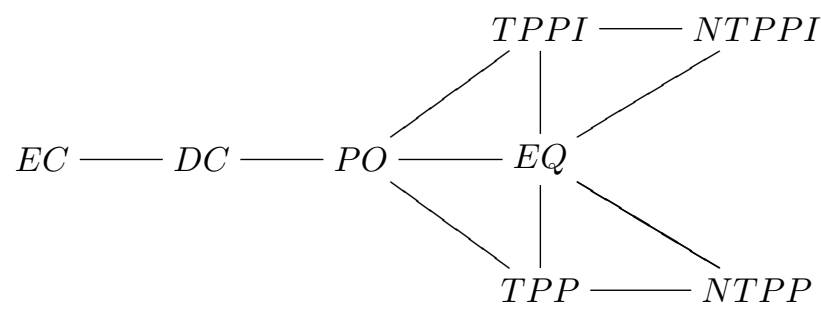

Fig. 2. A neighborhood graph for RCC8

the empty relation by the inference rules (1) and (2).

We say a scenario $s_{N}$ is a scenario of a $Q C N N$ iff every base relation $b_{i j}$ in $s_{N}$ is a subset of the relation $R_{i j}$ in $N$.

Definition 6 (Consistency). Let $N$ be a $Q C N . N$ is said to be consistent iff it admits at least one scenario.

The set of all scenarios of $N$ is denoted by $[N]$. The relations holding between $v_{i}$ and $v_{j}$ in $N$ are denoted by $N[i, j]$. These concepts also motivate the de-facto standard way of deciding consistency of QCNs by applying constraint programming to find some scenario using backtracking search while applying the inference rules to establish local consistency during search [WW09]. This is also the basis for the qualitative constraint solver GQR [WWG09] which utilizes optimized constraint propagation algorithms combined with heuristic backtracking search.

\section{B. Merging Qualitative Constraints Networks}

We briefly recall concepts of merging information in the context of QCNs as detailed in recent works [WD10], [CKMS10]. Some of these works even propose a framework for merging heterogeneous QCNs [CKMS09a], but in this paper we will only consider revising beliefs according to QCNs expressed over the same calculus. An extension to heterogeneous formalisms is possible but not our focus here.

Similar to the general case, merging a multi-set of QCNs $\mathcal{N}=\left\{N_{1}, \ldots, N_{n}\right\}$, called a belief profile, consists in providing a QCN which represents the information in the sources $N_{i}$. The merging operation can be constrained by a QCN denoted by $I C$ which represents absolute constraints that have to be respected by the solution. There can be no such constraints and in this case we consider that $I C=\top$. The result of the merging operation is denoted $\Delta_{I C}(\mathcal{N})$ possibly with decoration.

Merging operators can be of two types. These merging operators are based on the distance between solutions of each source (called semantic operators) or based on the number of violated constraints (called syntactic operators). The semantic merging operations first compute the distance between a scenario and QCNs, these distance are then aggregated in order to obtain a distance between a scenario and the whole belief profile. The syntactic operations compute the distance between a scenario and all constraints between two variables expressed in the profile before aggregating those distances. The solution then is the set of scenarios closest to $\mathcal{N}$.

An aggregation function is a function that associates to every finite tuple of non-negative integers a non-negative integer and which respects non-decreasingness, minimality and identity (as in [KLM04]). Known examples of aggregation functions are sum or average functions.

1) Distances: In the general case, both Wallgrün and Condotta et al. use a drastic distance (or Hamming distance) between base relations.

Definition 7 (Drastic distance between base relations). Let $b, b^{\prime}$ be base relations.

$$
d_{D}\left(b, b^{\prime}\right)=\left\{\begin{array}{rr}
0 & \text { if } b=b^{\prime} \\
1 & \text { otherwise }
\end{array}\right.
$$

An alternative is to consider a more fine-grained distance based on proximity of the base relations in the conceptual neighborhood graph [CKS08].

Definition 8 (Neighborhood distance between base relations). Let $N G$ be a conceptual neighborhood graph on elements of $B$ and $b, b^{\prime}$ base relations. We define the conceptual neighborhood distance

$$
d_{N G}\left(b, b^{\prime}\right)=\text { length of shortest path } b \text { to } b^{\prime} \text { in } N G
$$

Finally, the distance between scenarios $s, s^{\prime}$ is defined as the sum of the distances between every pair of corresponding constraints $\left(b_{i j}, b_{i j}^{\prime}\right)$ where $b_{i j} \in s$ and $b_{i j}^{\prime} \in s^{\prime}$.

2) Semantic merging operators $\Theta$ : The semantic operators return the set of the consistent scenarios of $I C$ which are the closest to $\mathcal{N}$ with respect to a given distance function. They are characterized by a pair $\left(d_{X}, f\right)$ where $d_{X}$ is a distance function between scenarios and $f$ is an aggregation function.

Definition 9 (Distance between a scenario and a QCN). Let $\mathcal{N}$ be a belief profile, $N$ be a $Q C N, s$ be a scenario of $N$ and $d_{X}$ be a distance function, then the distance between $a$ scenario and a $Q C N$ is defined as:

$d_{X}^{\Theta}(s, N)=\left\{\begin{aligned} \min \left\{d_{X}\left(s, s^{\prime}\right) \mid s^{\prime} \in[N]\right\} & \text { if } N \text { is consistent } \\ 0 & \text { otherwise }\end{aligned}\right.$

Thus the distance between a scenario and a belief profile according to the aggregation function $f$ is defined as:

$$
d_{X, f}^{\Theta}(s, \mathcal{N})=f\left\{d_{X}^{\Theta}(s, N) \mid N \in \mathcal{N}\right\}
$$

Definition 10 ( $\Theta$ merging operation). Let $\mathcal{N}$ be a belief profile, $I C$ be a $Q C N$ representing constraints, $f$ be an aggregation function, $s$ be a scenario of $\mathcal{N}$ and $d_{X}$ be a distance function, then the semantic merging operation is defined as:

$$
\begin{aligned}
& \Theta_{I C}^{X, f}(\mathcal{N})=\left\{s \in[I C] \mid \nexists s^{\prime} \in[I C]\right. \text { s.t. } \\
& \left.d_{X, f}^{\Theta}\left(s^{\prime}, \mathcal{N}\right)<d_{X, f}^{\Theta}(s, \mathcal{N})\right\}
\end{aligned}
$$

3) Syntactic merging operator $\Delta_{2}$ : Another way to define QCN merging operators is to compute for every pair $\left(v_{i}, v_{j}\right)$ the distance between a relation and the set of constraints holding between $v_{i}$ and $v_{j}$ in the belief profile $\mathcal{N}$. Then these distances are aggregated over every pair of variables. Each operator is characterized by a triple $\left(d_{X}, f, g\right)$ where $d_{X}$ is a distance between scenarios and $f$ and $g$ are aggregation functions.

Definition 11. Let $\mathcal{N}$ be a belief profile, $N$ be a $Q C N, v_{i}$ and $v_{j}$ be two variables of $\mathcal{N}, f$ and $g$ be two aggregation 
functions, $b$ be a base relation of $B$ and $d_{X}$ be a distance function, then the distance between a base relation and a pair of variables in $\mathcal{N}$ is defined as:

$$
d_{X, f}(b, \mathcal{N}[i, j])=f\left\{d_{X, B}(b, N[i, j]) \mid N \in \mathcal{N}\right\}
$$

where

$$
d_{X, B}(b, R)=\min \left\{d_{X}\left(b, b^{\prime}\right) \mid b^{\prime} \in R\right\}
$$

Thus the distance between a scenario and a belief profile is defined as:

$d_{X, g, f}^{\Delta_{2}}(s, \mathcal{N})=g\left\{d_{X, f}\left(s_{i j}, \mathcal{N}[i, j]\right) \mid\left(v_{i}, v_{j}\right) \in V^{2}\right.$ and $\left.i<j\right\}$

Definition 12 ( $\Delta_{2}$ merging operation). Let $\mathcal{N}$ be a belief profile, $I C$ be a $Q C N$ representing constraints, $s$ be a scenario of $\mathcal{N}, f$ and $g$ be two aggregation functions, $b$ be a base relation of $B$ and $d_{X}$ be a distance function, then the semantic merging operation is defined as:

$$
\begin{aligned}
& \Delta_{2, I C}^{X, g, f}(\mathcal{N})=\left\{\quad s \in[I C] \mid \nexists s^{\prime} \in[I C]:\right. \\
& \left.d_{X, g, f}^{\Delta_{2}}\left(s^{\prime}, \mathcal{N}\right)<d_{X, g, f}^{\Delta_{2}}(s, \mathcal{N})\right\}
\end{aligned}
$$

For more details, see [CKMS10].

\section{Belief Revision and AGM postulates}

Belief revision is an operation describing what happens to the belief of an agent when he learns more reliable information about the state of the world. Let $K$ be a belief base and $A$ be a formula, then the revision of $K$ by $A$ is denoted by $K \circ A$. The result of a revision operation should resolve the conflicts, be consistent with the new information and be as close as possible to the old belief state.

In order to evaluate the revision operations Alchourròn, Gärdenfors and Makinson introduced postulates in [AGM85] for characterizing good behaviors of revision operators. These postulates were later revised by Katsuno and Mendelzon [KM91].

Definition 13 (AGM Postulates). Let $K$ be a belief base and $A, B$ be formulas, then the belief base $K \circ A$ is such that:

(KM1) $K \circ A \vdash A$.

(KM2) If $K \wedge A$ is satisfiable, then $K \circ A \equiv K \wedge A$.

(KM3) If $A$ is satisfiable, then $K \circ A$ is also satisfiable.

(KM4) If $K_{1} \equiv K_{2}$ and $A_{1} \equiv A_{2}$, then $K_{1} \circ A_{1} \equiv K_{2} \circ A_{2}$

(KM5) $(K \circ A) \wedge B \vdash K \circ(A \wedge B)$

(KM6) If $(K \circ A) \wedge B$ is satisfiable, then

$(K \circ A) \wedge B \vdash(K \circ A) \wedge B$

The meaning of these postulates are the following. (KM1) ensures that the new information is present in the result of revision. (KM2) and (KM3) states that if the new information is not contradictory with the old one, then the result of revision is just their conjunction. (KM4) is the principle of irrelevance of syntax. (KM5) and (KM6) are called additional postulates, and states the principle of minimality of change.

\section{REVISION OF QUALitative CONSTRAINT NeTwORKS}

\section{A. From merging to revision operations}

The operations we define in this paper are inspired by the qualitative merging operations found in the related work described supra. The idea is to consider the operation $K \circ A$ of revising $K$ by $A$ as a special case of merging where $K$ is merged under the constraint $A$, i.e., $\Delta_{A}(K)$. We adapted both the semantic and the syntactic operators. We first give their definition before a short study about their properties relatively to the AGM postulates.

The semantic revision operation, denoted by $\circ_{X, \Theta}$, provides as a result the scenarios of $A$ which are the closest to the scenarios of $K$. Formally, this is defined as follows:

Definition $14\left(\circ_{X, \Theta}\right.$ revision operator). Let $K$ and $A$ be two $Q C N s, s$ and $s^{\prime}$ be two consistent scenarios and $d_{X}$ a distance function. The semantic revision operation of $K$ by $A$, denoted by $K \circ_{X, \Theta} A$, is defined as:

$K \circ_{X, \Theta} A=\left\{s \in[A] \mid \nexists s^{\prime} \in[A]\right.$ s.t. $\left.d_{X}^{\Theta}\left(s^{\prime}, K\right)<d_{X}^{\Theta}(s, K)\right\}$

Example 1. Lets consider the following example with $K=\{(a, T P P I, b)(a, E Q N T P P D C, c)(a, N T P P I, d)$ $(b, N T P P \quad P O, c)\}$ and $A=\{(a, N T P P I, b)$ $(a, P O \quad D C, d) \quad(b, P O, c) \quad(c, D C \quad E C, d)\}$. Please note that the relations which are not explicitly specified here are considered to be the universal relations. There are 22 scenarios for $A$ and 3 scenarios of $K$.

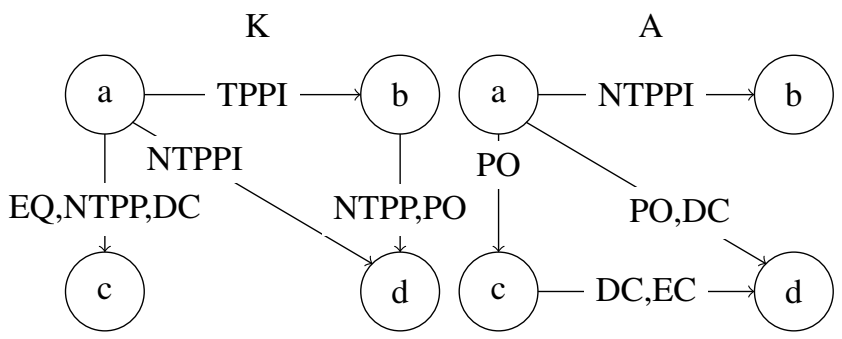

Fig. 3. Qualitative Constraint Networks $K$ and $A$ from Example 1

For $K \circ_{X, \Theta} A$ with a neighborhood distance, there is only one scenario of $A$ which is minimal wrt. its distance to $K$. The result of $K \circ_{N G, \Theta} A$ is thus: $K \circ_{N G, \Theta} A=$ $\{s=\{(a, N T P P I, b)(a, P O, c)(a, P O, d)(b, P O, c)$ $(b, P O, d)(c, D C, d)\}\}$ whose closest scenario from $K$ is $s^{\prime}=\{(a, T P P I, b)(a, D C, c)(a, N T P P I, d)(b, D C, c)$ $(b, P O, d)(c, D C, d)\}$ (The distance between $s$ and $s^{\prime}$ thus is $1+1+2+1+0+0=5)$.

The syntactic revision operation, denoted by $\circ_{X, \Delta_{2}}$, provides as a result the scenarios of $A$ which are the closest to the constraints given in $K$. This operation is defined as:

Definition $15\left(\circ_{X, \Delta_{2}}\right.$ revision operator). Let $K$ and $A$ be two $Q C N s, s$ and $s^{\prime}$ be two consistent scenarios and $d_{X}$ a distance function. The syntactic revision operation of $K$ by $A$, denoted by $K \circ_{X, \Delta_{2}} A$, is defined as:

$K \circ_{X, \Delta_{2}} A=\left\{s \in[A] \mid \nexists s^{\prime} \in[A]\right.$ s.t. $\left.d_{X}^{\Delta_{2}}\left(s^{\prime}, K\right)<d_{X}^{\Delta_{2}}(s, K)\right\}$

where $d_{X}^{\Delta_{2}}(s, K)=\sum_{v_{i}, v_{j} \in V^{2}} d_{X}\left(b_{i j}, K[i, j]\right)$. 
These operations take networks as input but the result is given in the form of a set of scenarios. From a purely theoretical point of view, this would be a situation to be avoided and one would expect the result and the inputs to be in the same form. One solution might be to provide a minimal network which generates all of the scenarios in the solutions like in [WD10]. From a practical point of view, this solution has two major drawbacks: first on some instances (particularly when using the neighborhood distance) the resulting graph may be bigger than the input, second this new constraint network may have scenarios which were not scenarios of $K \circ A$. There is a trade-off here between discontinuity in the knowledge representation and a loss of information.

1) Formal properties of the operators: We now provide a study of both our syntactic and semantic revision operations with respect to the AGM postulates.

Proposition 1. For $d_{X}$ being either $d_{D}$ or $d_{N G}$, the $\circ_{X, \Theta}$ revision operation respects the (KM1), (KM2), (KM3), (KM4), (KM5) and (KM6) postulates.

Proof:

(KM1) If $s \in\left[K \circ_{X, \Theta} A\right]$, then by definition $s \in[A]$. Thus, $K \circ_{X, \Theta} A \vdash A$.

(KM2) If $K \wedge A$ is consistent, then all consistent scenario $s$ such that $s \in[A]$ and $s \in[K]$.

$K \circ A \subseteq K \wedge A$. We assume $s \in K \circ A$, then $s \in[A]$ and $s \in \min \left(d^{\Theta}(s, K)\right)$. As we know, there exist scenarios which are models of both $A$ and $K$, then $d_{X}^{\Theta}(s, K)=0$. Thus, $s \in[A]$ and $s \in[K]$. Thus $s \in[K \wedge A]$.

$K \wedge A \subseteq K \circ A$. We assume $s \in[K \wedge A]$. Thus, $s \in[A]$ and $s \in[K]$. Then $s \in[A]$ and $s \in \min \left(d_{X}^{\Theta}(s, K)\right)$ and $s \in K \circ A$. (KM3) The solutions of $K \circ_{X, \Theta} A$ is, by definition, a set of consistent scenarios. Thus, it is consistent.

(KM4) is trivial according to the definition of $\circ_{X, \Theta}$.

(KM5) If $\left(K \circ_{X, \Theta} A\right) \wedge B$ is inconsistent, the result is trivial. Otherwise, there exists at least a consistent scenario $s$ where $s \in[K \circ A]$ (and thus $s \in[A]$ ) and $s \in[B]$. Now, we assume there exists a consistent scenario $s^{\prime}$ where $s^{\prime} \in\left[K \circ_{X, \Theta}(A \wedge\right.$ $B)]$ and $s \notin\left[K \circ_{X, \Theta}(A \wedge B)\right]$. We know that $s \in[A]$ and $s \in[B]$, thus $d_{X}^{\Theta}\left(s^{\prime}, K\right)<d_{X}^{\Theta}(s, K)$ which is impossible because $s^{\prime}$ would have been a scenario of $\left(K \circ_{X, \Theta} A\right) \wedge B$.

(KM6) We assume that there exists a consistent scenario $s$ such that $s \in\left(K \circ_{X, \Theta} A\right) \wedge B$ and $s \notin K \circ_{X, \Theta}(A \wedge B)$. That means, it exists a consistent scenario $s^{\prime}$ such that $s^{\prime} \in[A \wedge B]$ and $d_{X}^{\Theta}\left(s^{\prime}, K\right)<d_{X}^{\Theta}(s, K)$. But, in that case $s^{\prime}$ would have been a scenario of $\left(K \circ_{X, \Theta} A\right) \wedge B$ and thus $s$ could not have been one which is impossible.

Proposition 2. For $d_{X}$ being either $d_{D}$ or $d_{N G}$, the $\circ_{X, \Delta_{2}}$ revision operation respects the (KM1), (KM2), (KM3), (KM5) and (KM6) postulates.

Proof:

(KM1), (KM2), (KM3), (KM5) and (KM6). For these postulates, the proofs are similar to the Proposition 1.

(KM4) $A_{1} \equiv A_{2}$ means that the set of consistent scenarios of $A_{1}$ is exactly those of $A_{2}$. Counter-example in RCC5 Calculus for the neighborhood distance (a counter-example can also be found for the drastic distance):
$A=\left\{\left(v_{1} E Q v_{2}\right),\left(v_{1} E Q, P P v_{3}\right),\left(v_{2} E Q, P P v_{3}\right)\right\}$

$K_{1}=\left\{\left(v_{1} P C v_{2}\right),\left(v_{1} P P, P C v_{3}\right),\left(v_{2} P C v_{3}\right)\right\}$

$K_{2}=\left\{\left(v_{1} P C v_{2}\right),\left(v_{1} P C v_{3}\right),\left(v_{2} P C v_{3}\right)\right\}$

In this case, $d_{N G}^{\Delta_{2}}\left(s_{1}, K_{1}\right)=1+0+2=3, d_{N G}^{\Delta_{2}}\left(s_{2}, K_{1}\right)=1+$ $1+1=3, d_{N G}^{\Delta_{2}}\left(s_{1}, K_{2}\right)=1+1+2=4$ and $d_{N G}^{\Delta_{2}}\left(s_{2}, K_{2}\right)=$ $1+1+1=3$.

We thus have $K_{1} \circ_{N G, \Delta_{2}} A=\left\{\left\{\left(v_{1} E Q v_{2}\right),\left(\begin{array}{lll}v_{1} & P P & v_{3}\end{array}\right)\right.\right.$, $\left.\left.\left(v_{2} P P v_{3}\right)\right\},\left\{\left(\begin{array}{lll}v_{1} & E Q & v_{2}\end{array}\right),\left(v_{1} E Q v_{3}\right),\left(v_{2} E Q v_{3}\right)\right\}\right\}$ and $K_{2} \circ_{N G, \Delta_{2}} A=\left\{\left\{\left(v_{1} E Q v_{2}\right),\left(v_{1} P P v_{3}\right),\left(v_{2} P P v_{3}\right)\right\}\right\}$. Thus $K_{1} \circ_{N G, \Delta_{2}} A \not \equiv K_{2} \circ_{N G, \Delta_{2}} A$.

The syntactic operation does not respect the (KM4) postulate. This is due to the fact that it measures the distance between the scenario of $\mathrm{A}$ and the constraints in $K$. The $\circ_{X, \Delta_{2}}$ operation is thus attached to syntax of $K$. This is not to be considered as a drawback as it is an intended feature of syntactic operations to take syntax into account.

In [JT07], Jin and Thielscher defined a weaker version of the (KM4) postulate. Namely, the weak syntactic irrelevance postulate, denoted by (KM4'), is the following:

(KM4') If $A_{1} \equiv A_{2}$, then $K \circ A_{1} \equiv K \circ A_{2}$.

Proposition 3. For $d_{X}$ being either $d_{D}$ or $d_{N G}$, the $\circ_{X, \Delta_{2}}$ and $\circ_{X, \Theta}$ revision operations respect the (KM4') postulate.

Thus, the syntactic revision operations respect almost all postulates and all postulates if we accept their weaker version. On the other hand, the semantic operation respects all the AGM postulates.

\section{An IMPLEMENTATION BASED ON GQR}

In order to perform the revision operation, we here propose an adaptation of the depth-first search algorithm usually employed in checking the consistency of qualitative constraint networks. More specifically, the revision operations can be handled with a branch-and-bound approach. We implemented our algorithms in the qualitative constraint solver GQR, thus taking advantage of fast computations of inference rules and the existing 2-way branching code with heuristics. Originally, the search algorithm terminates once a scenario has been found, so we have modified it into a branch-and-bound approach that computes distances according to some distance function $d$ on two QCNs and keeps iterating until no better scenarios can be computed. For this it keeps track of the best scenarios and the best distance encountered so far and prunes the tree according to this information.

A direct search for the solution to a revision problem requires us to explore the whole search space in order to compute all optimal scenarios, which is costly. On the other hand, searching for just one scenario with a given distance can be stopped as soon as such a scenario is found. For this reason, we search for the minimal distance prior to the computation of all scenarios with this optimal distance. To compute the minimal distance, we use a dichotomy search. Here, we maintain and revise an interval $[\min , \max ]$ that includes the optimal distance. We can easily estimate the maximum distance for both $d_{D}$ and $d_{N G}$ to initialize this interval. We run the branch-and-bound algorithm with the mean of $\min , \max$ as the bound and abort as soon as a 
scenario with at maximum this bound has been found. Such a scenario (or the result that none exists) than refines our interval, and we can continue with another branch-and-bound search until we have proven the minimal bound. Algorithm 1 gives the pseudo code for this approach.

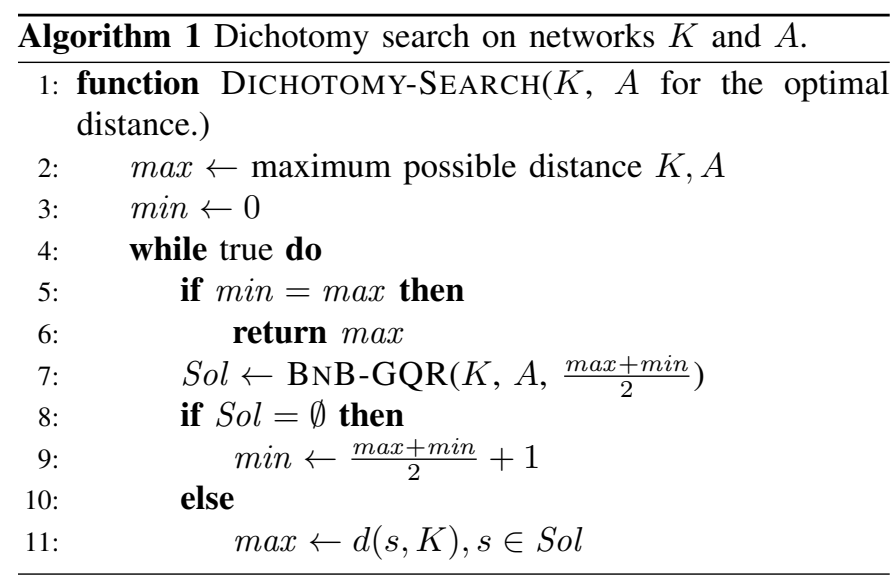

Algorithm 2 gives pseudo code for the branch-and-bound search within GQR. A slight variation of Algorithm 2 that returns the first found scenario is used for the initial dichotomy search of the minimal distance.

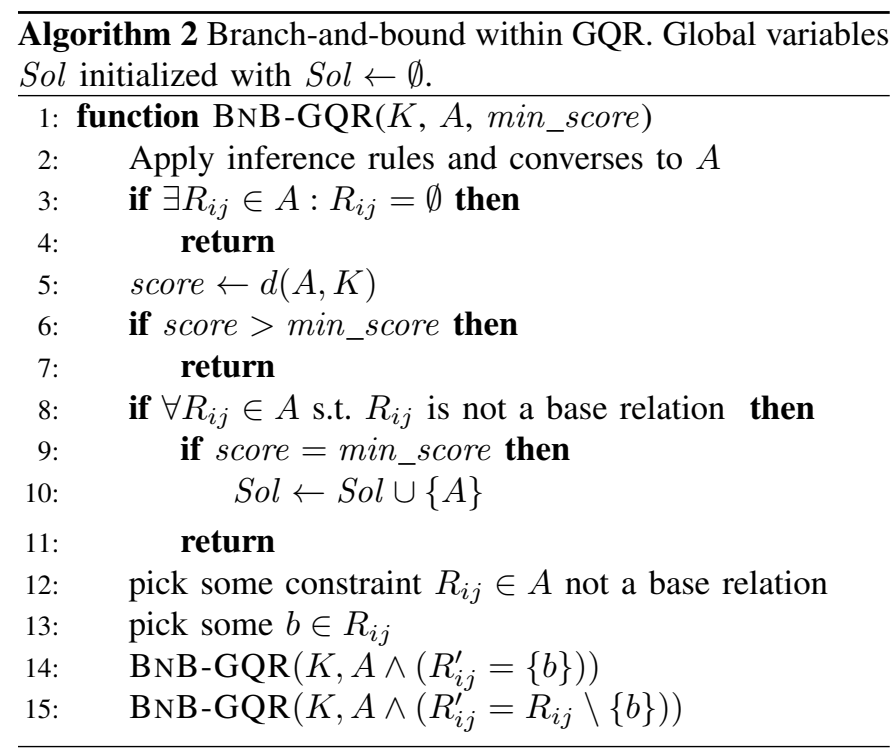

In Algorithm 2 we can set $d$ to be

$$
d\left(N, N^{\prime}\right)=\sum_{i, j} \min \left\{d_{X}\left(b, C_{i j}^{\prime}\right) \mid b \in C_{i j}\right\}
$$

for $d_{X}$ either $d_{D}$ or $d_{N G}$, to compute syntactic revision $\circ_{\Delta_{2}}$. In order to allow for a simple and space-efficient computation of semantic revision $\circ_{\Theta}$, we set:

$$
d\left(N, N^{\prime}\right)=\operatorname{BNB}-\mathrm{GQR}\left(N, N^{\prime}, \text { min_score }\right)
$$

where this invocation of BNB-GQR uses either $d_{D}$ or $d_{N G}$ as distance function and the current bound min_score to prune the search space. It further does not need to compute more than one scenario per current score.

\section{A. Complexity Result}

The complexity of our algorithm is what one can expect when comparing with revision in propositional logic (see [EG92]).

Proposition 4. For $d_{X}$ being either $d_{D}$ or $d_{N G}$, determining if $K \circ_{X, \Delta_{2}} A \models B$ or $K \circ_{X, \Theta} A \models B$ is in $\mathcal{O}\left(N P^{\log \left(n^{2}\right)}\right)$ where $n$ is the number of variables.

This result directly follows from the algorithm. The dichotomic search requires $\log \left(n^{2}\right)$ calls to find the size of the optimal scenarios then finding all scenarios of that size is one additional call.

\section{EXPERIMENTAL STUDY}

We ran an experimental evaluation of the proposed revision and distance functions. For this we have focused on the RCC8 calculus as it is one of the most well-known calculi with applications in Geo-Information Systems. Our experiments were run on an AMD Opteron $2.3 \mathrm{GHz}$ with $4 \mathrm{~GB}$ of memory and a time limit of 2 hours. Due to a lack of real world data we use randomly generated benchmark instances according to two models. Both models rely on random constraint graphs, taking the graphs size and degree as parameters.

The first model we use is the $A^{\prime}$-model that generates graphs with $n$ nodes and a degree $d$. To form QCNs the edges in these graphs are labeled with random relations drawn from $2^{B}$ without the empty relation. In particular, the degree parameter $d$ controls how constrained the instances are. With a low degree instances are under-constrained and most likely satisfiable. Similarly, with a high degree instances are overconstrained and thus most likely unsatisfiable. As usual we can use the parameter $d$ to generate instances from the phase transition. This is the de-facto standard model for QCNs, used e.g., in [WW09]. We here restrict ourselves to satisfiable instances only, that is, generated instances that are inconsistent are discarded upon generation.

As an alternative second model we consider extracting a scenario $s$ with $n$ nodes from a hard problem instance (e.g., an $A^{\prime}$ model instance from the phase transition region) and then overlaying it with an instance $a$ from the $A^{\prime}$ model - also with $n$ nodes - in the following way: if there is no relation between variables $v_{i}$ and $v_{j}$ in $a$ we use the relation in $s$; if there is a relation between variables $v_{i}$ and $v_{j}$ in $a$ we use the union of this relation with the corresponding relation in $s$. The instance $a$ thus acts like noise on top of the scenario $s$. Instances from this model can be seen as noisy input and we denote this model by $N$. The degree of $N$ is the degree of the used $a$ from the $A^{\prime}$ model.

It should be noted here that the size parameter $n$ corresponds to the number of variables and thus the number of relations is $n^{2}$. The number of relations is however more important as the number of possible scenarios in these models is bounded by $O\left(8^{n^{2}-d \cdot n} \cdot 4^{d \cdot n}\right)=O\left(2^{\left(n^{2}\right)}\right)$.

For each considered setup (model, size, degree) we generated 1000 pairs of random networks to conduct our revision experiments. We then ran the suggested algorithm for syntactic and semantic revision with both given distance functions. 
For the $A^{\prime}$ model we present in Fig. 4, Fig. 5, and Fig. 6 the running times (in seconds), the average distances of the best scenarios and the number of scenarios in the solutions for QCNs of size 10 with different degrees. Please note here that a constraint network of size 10 has 45 non-trivial relations. With these random instances $K \wedge A$ was never consistent.

\begin{tabular}{c|r|r|r|r}
\hline Revision & ${ }^{\circ}{ }_{D, \Delta_{2}}$ & ${ }^{\circ} N G, \Delta_{2}$ & ${ }^{\circ} D, \Theta$ & ${ } N G, \Theta$ \\
\hline avg. time & 2.244 & 1.026 & 54.696 & 9.079 \\
std. dev & 7.699 & 3.790 & 183.168 & 32.414 \\
\hline optimal bound & 8.326 & 9.867 & 14.066 & 20.887 \\
\hline avg. scen. & 562452 & 174399 & 61853 & 6444 \\
std. dev & 1976716 & 695716 & 238899 & 33332 \\
\hline
\end{tabular}

Fig. 4. Running times (s), minimal distance of solutions and number of scenarios in the solutions for $A^{\prime}$ with size 10 and degree 7 .

\begin{tabular}{c|r|r|r|r}
\hline Revision & ${ }^{\circ} D, \Delta_{2}$ & ${ }^{\circ} N G, \Delta_{2}$ & ${ }^{\circ} D, \Theta$ & ${ } N G, \Theta$ \\
\hline avg. time & 0.235 & 0.120 & 10.961 & 1.535 \\
std. dev & 0.761 & 0.348 & 33.953 & 2.835 \\
\hline optimal bound & 10.865 & 12.924 & 17.446 & 26.374 \\
\hline avg. scen. & 46757 & 13603 & 10988 & 343 \\
std. dev & 173028 & 50817 & 35531 & 1330 \\
\hline
\end{tabular}

Fig. 5. Running times (s), minimal distance of solutions and number of scenarios in the solutions for $A^{\prime}$ with size 10 and degree 8 .

\begin{tabular}{c|r|r|r|r}
\hline Revision & ${ }^{\circ},_{2}$ & ${ }^{\circ}{ }_{N G, \Delta_{2}}$ & ${ }^{\circ}, \Theta$ & ${ }$ \\
\hline avg. time & 0.030 & 0.019 & 3.404 & 0.550 \\
std. dev & 0.066 & 0.030 & 9.834 & 0.735 \\
\hline optimal bound & 13.626 & 16.322 & 20.857 & 32.593 \\
\hline avg. scen. & 3624 & 863 & 4030 & 45 \\
std. dev & 10416 & 2174 & 13478 & 96 \\
\hline
\end{tabular}

Fig. 6. Running times (s), minimal distance of solutions and number of scenarios in the solutions for $A^{\prime}$ with size 10 and degree 9.

For the $A^{\prime}$ model, we can observe two interesting results. With semantic revision we almost always get drastically less scenarios in a solution than with syntactic revision, as semantic revision is more fine-grained when it comes to differences between a scenario and a network. However, we can also see that syntactic revision is always faster here even though there are far more scenarios to be computed. Further, these results highlight that the distance functions have a severe impact on the efficiency of the revision operation. The drastic distance seems rather impractical here as it leads to larger solutions and worse runtime.

As expected, the running times are high compared to standard satisfiability tests for QCNs where sizes of more than 100 nodes are common. This may be explained by several factors: firstly it is necessary to calculate all optimal scenarios as opposed to satisfiability test that terminate on the first scenario, secondly the total number of scenarios in instances from the $A^{\prime}$ model are extremely high (for a degree of 8 we have seen instances with more than 1.3 billion scenarios and there are even more scenarios in instances with lower degrees).

As can be seen from our results the problem caused by the number of scenarios in solutions tends to disappear when the degree of the networks increases and thus the networks are tighter and have less scenarios in total (see Fig. 7 for an overview of runtimes according to degree). While this is a problem with the $A^{\prime}$ model in particular, it is of course also

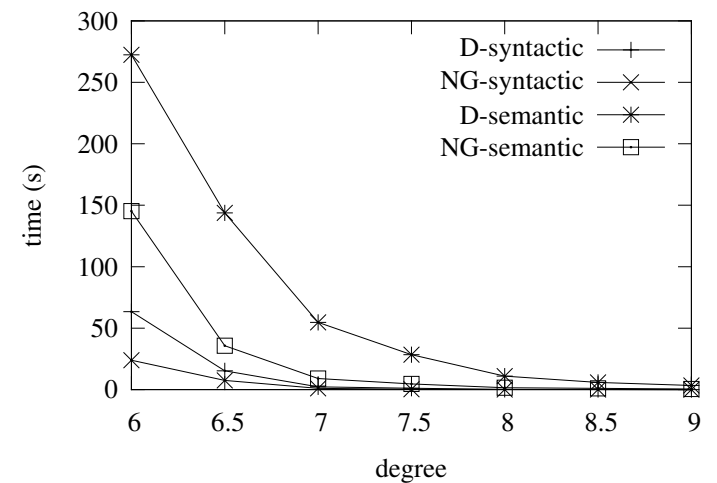

Fig. 7. Overview of running times (s) according to degree for $A^{\prime}$ model with 10 nodes.

the case that such instances can appear in practice making revision of QCNs very hard. We did not expect such issues and as far as we know we are the first to discuss this very important issue. Given the number of scenarios encountered here, turning towards $A^{\prime}$ instances with more nodes seems infeasible for revision as there will be far too many scenarios (in particular with drastic distances).

As the $A^{\prime}$ model has proven troublesome we also consider the $N$ model. This model has in general less scenarios than the $A^{\prime}$ model for low degrees, as low degree here implies more base relations from a scenario appear in the input. This allows us to scale the number of nodes without introducing too many scenarios. We present in Fig. 8, Fig. 9, and Fig. 10 the running times (in seconds), the average distances of the best scenarios and the number of scenarios in the solution set for instances of the $N$ model with 25 nodes and degree 10, 50 nodes and degree 5 , and 50 nodes and degree 8 .

For the $N$-model there are much fewer scenarios with all approaches. The general trends observed with the $A$-model apply here as well. However, the difference in the number of scenarios in the solution for drastic and neighborhood graph distance is far more distinct than before. We conjecture this is due to the lower number of scenarios in the instances which makes the neighborhood distance more discriminative.

Unlike with the $A^{\prime}$ model were all problems were solved by

\begin{tabular}{c|r|r|r|r}
\hline Revision & ${ }^{\circ} D, \Delta_{2}$ & ${ }^{\circ} N G, \Delta_{2}$ & ${ }^{\circ}, \Theta$ & ${ } N G, \Theta$ \\
\hline avg. time & 702.640 & 7.277 & 1711.857 & 102.412 \\
std. dev & 1284.515 & 24.426 & 1752.225 & 298.782 \\
\hline optimal bound & 127.415 & 238.376 & 147.895 & 302.010 \\
\hline avg. scen. & 47654662 & 102835 & 283818 & 221 \\
std. dev & 97586857 & 392583 & 405064 & 719 \\
\hline fail. (\%) & 18.2 & 0.1 & 58.5 & 0.6 \\
\hline
\end{tabular}

Fig. 8. Running times (s), minimal distance of solutions and number of scenarios in the solutions for $N$ with size 25 and degree 10 .

\begin{tabular}{c|r|r|r|r}
\hline Revision & ${ }^{\circ},_{\Delta_{2}}$ & ${ }^{\circ} N, \Delta_{2}$ & ${ }^{\circ}{ }_{D, \Theta}$ & ${ } N G, \Theta$ \\
\hline avg. time & 3.244 & 0.048 & 291.703 & 1.695 \\
std. dev & 10.862 & 0.018 & 678.235 & 1.082 \\
\hline optimal bound & 571.524 & 1206.413 & 595.610 & 1279.487 \\
\hline avg. scen. & 60950 & 12 & 19795 & 3 \\
std. dev & 244464 & 23 & 54635 & 3 \\
\hline fail. (\%) & 0 & 0 & 1.6 & 0 \\
\hline
\end{tabular}

Fig. 9. Running times (s), minimal distance of solutions and number of scenarios in the solutions for $N$ with size 50 and degree 5 . 


\begin{tabular}{c|r|r|r|r}
\hline Revision & ${ }^{\circ} \Delta_{2}$ & ${ }^{\circ} N G, \Delta_{2}$ & ${ }^{D}, \Theta$ & ${ } N G, \Theta$ \\
\hline avg. time & 521.108 & 0.240 & 1869.782 & 12.283 \\
std. dev & 1082.643 & 0.393 & 1925.819 & 15.778 \\
\hline optimal bound & 546.057 & 1136.532 & 580.125 & 1250.131 \\
\hline avg. scen. & 8222188 & 288 & 59852 & 11 \\
std. dev & 19319972 & 794 & 79558 & 18 \\
\hline fail. (\%) & 8.5 & 0 & 53 & 0 \\
\hline
\end{tabular}

Fig. 10. Running times (s), minimal distance of solution and number of scenarios in the solution for $N$ with size 50 and degree 8 .

all approaches, here many instances remained unsolved with the drastic distance (for numbers see the tables). As the $N$ model is closer to instances that can be expected in application scenarios, this underlines the importance of the neighborhood distance and its superiority to the drastic distance.

\section{CONCLUSION}

We proposed two novel revision operations for qualitative information based on the merging operators for such information introduced by Condotta et al.: a syntactic operation $\circ_{X, \Delta_{2}}$ and a semantic operation $\circ_{X, \Theta}$. These operations have been extensively studied and have shown good results relatively to the AGM postulates. Further, we have proposed new algorithms for these operators and discussed their complexity. These revision operations have been implemented and a first experimental study of this implementation has been provided. Our study, which seems to be the first in this line of research, highlights several shortcomings of the revision (and merging) operators that have been overlooked so far.

In particular, both revision operations show good theoretical behavior with respect to the AGM postulates, but nevertheless, the first results show that this task is extremely difficult to use in real applications. While solvers might be improved, the extremely high number of solutions in even small qualitative constraint networks will prevent any implementation to run on reasonably sized networks. This shows the definitions of merging operations are not yet satisfactory (the same problem will arise in the merging case especially for the operators involving the max-aggregation) and that, all the more, the straightforward definition of revision from those merging operations are not satisfactory. Maybe the use of selection functions within the revision process can help: as the number of solutions is extremely high, it may be useful to have a selection function that can a priori discard most of the solutions and thus save time. Our results further show shortcomings of the common distance functions, as even small input formulas can lead to far too many scenarios with the same optimal distance. More refined and discriminating distances are an interesting line of work.

It is also interesting to study how this revision implementation can be extended to the update problem and how it behaves in this context. The definition of update would restrict the change around the variable involved in the update process (e.g., if the relation between $v_{1}$ and $v_{2}$ is updated then the relation between $v_{5}$ and $v_{6}$ would remain unchanged in the update process while it may change during revision). Future work will also investigate more restrictive definitions in order to make implementations applicable to large scale scenarios.
Further, we also want to continue the work on analyzing postulates for classical revision operations on QCNs [Dos11].

\section{ACKNOWLEDGMENT}

We thank the anonymous reviewers for helpful comments. This work was supported by DFG (Transregional Collaborative Research Center SFB/TR 8 Spatial Cognition, project R4[LogoSpace]).

\section{REFERENCES}

[AGM85] Carlos E. Alchourrón, Peter Gärdenfors, and David Makinson On the logic of theory change: Partial meet contraction and revision functions. Journal of Symbolic Logic, 50(2):510-530, 1985.

[Ben94] Brandon Bennett. Spatial reasoning with propositional logics. In Proceeding of KR, pages 51-62, 1994.

[CH01] Anthony G. Cohn and Shyamanta M. Hazarika. Qualitative spatial representation and reasoning: An overview. Fundamenta Informaticae, 46(1-2):1-29, 2001.

[CKMS09a] Jean-François Condotta, Souhila Kaci, Pierre Marquis, and Nicolas Schwind. Merging qualitative constraint networks defined on different qualitative formalisms. In Proceedings of COSIT, pages 106-123, 2009.

[CKMS09b] Jean-François Condotta, Souhila Kaci, Pierre Marquis, and Nicolas Schwind. Merging qualitative constraints networks using propositional logic. In Proceedings of ECSQARU, pages 347-358, 2009.

[CKMS10] Jean-François Condotta, Souhila Kaci, Pierre Marquis, and Nicolas Schwind. A syntactical approach to qualitative constraint networks merging. In Proceedings of LPAR, pages 233 247, 2010.

[CKS08] Jean-François Condotta, Souhila Kaci, and Nicolas Schwind. A framework for merging qualitative constraints networks. In Proceedings of FLAIRS, pages 586-591, 2008.

[Dos11] André Doser. Revisionsoperatoren auf qualitativen Constraintnetzen, Bachelor's thesis, Department of Computer Science, Albert-Ludwigs-Universität Freiburg, 2011.

[EAT92] Max J. Egenhofer and Khaled K. Al-Taha. Reasoning about gradual changes of topological relationships. In Spatio-Temporal Reasoning, pages 196-219, 1992.

[EG92] Thomas Eiter and Georg Gottlob. On the complexity of propositional knowledge base revision, updates, and counterfactuals. In Proceedings of PODS, pages 261-273, 1992.

[JT07] Yi Jin and Michael Thielscher. Iterated belief revision, revised. Artificial Intelligence, 171(1):1-18, 2007.

[KLM04] Sébastien Konieczny, Jérôme Lang, and Pierre Marquis. Da2 merging operators. Artificial Intelligence Journal, 157(1-2):4979, 2004.

[KM91] Hirofumi Katsuno and Alberto O. Mendelzon. On the difference between updating a knowledge base and revising it. In Proceedings of KR, pages 387-394, 1991.

[RC89] David A. Randell and Anthony G. Cohn. Modelling topological and metrical properties in physical processes. In Proceeding of $K R$, pages 55-66, 1989.

[RCC92] David A. Randell, Zhan Cui, and Anthony G. Cohn. A spatial logic based on regions and connection. In Proceeding of $K R$, pages 165-176, 1992.

[Ren02] Jochen Renz. Qualitative Spatial Reasoning with Topological Information, volume 2293 of LNCS. Springer, 2002.

[Wal10] Jan Oliver Wallgrün. Qualitative spatial reasoning for topological map learning. Spatial Cognition \& Computation, 10(4):207246, 2010.

[WD10] Jan Oliver Wallgrün and Frank Dylla. A relation-based merging operator for qualitative spatial data integration and conflict resolution. Technical Report 022-06/2010, SFB/TR 8 Spatial Cognition; http://www.sfbtr8.uni-bremen.de/, 2010.

[WW09] Matthias Westphal and Stefan Wölfl. Qualitative CSP, finite CSP, and SAT: Comparing methods for qualitative constraintbased reasoning. In Proceedings of IJCAI, pages 628-633, 2009.

[WWG09] Matthias Westphal, Stefan Wölfl, and Zeno Gantner. GQR: A fast solver for binary qualitative constraint networks. In $A A A I$ Spring Symposium: Benchmarking of Qualitative Spatial and Temporal Reasoning Systems, pages 51-52, 2009. 